\title{
High-Gd-Payload P22 protein cage nanoparticles for imaging vascular inflammation
}

\author{
Hisanori Kosuge ${ }^{1 *}$, Masaki Uchida², Janice Lucon², Shefah Qazi ${ }^{2}$, Trevor Douglas ${ }^{2}$, Michael V McConnell ${ }^{1}$ \\ From 16th Annual SCMR Scientific Sessions \\ San Francisco, CA, USA. 31 January - 3 February 2013
}

\section{Background}

The bacteriophage P22 protein cage can be bioengineered to contain a high-relaxivity gadolinium (Gd) payload internally and targeting ligands externally. It also enables phage-library-based identification of novel targets. Thus, P22 may have advantages for molecular/cellular imaging by MRI.

\section{Methods}

1) P22: The P22 protein cage $(60 \mathrm{~nm})$ is bioengineered with an internal polymer network with amine functional groups allowing incorporation of $~ 9100 \mathrm{Gd}-\mathrm{DTPA}$ molecules per cage via the amine groups (Figure 1: [1]). This provides a per cage relaxivity of $70000 \mathrm{mM}^{-1} \mathrm{~s}^{-1}$, superior to Gd-DTPA for the equivalent Gd concentration.

2) Atherosclerosis Models: Both ApoE-deficient $\left(\mathrm{ApoE}^{-/}\right)$and FVB mice were used. ApoE ${ }^{-/-}$mice develop atherosclerosis enhanced by high-fat diet. FVB mice develop macrophage-rich carotid lesions with carotid ligation in combination with high-fat diet and diabetes induction [2].

3) P22-polymer-Gd in vivo MR imaging: Mice were injected intravenously with P22-polymer-Gd $(\mathrm{N}=5,20$ $\mu \mathrm{mol} \mathrm{Gd} / \mathrm{kg}$, one-fifth the typical clinical dose) or Magnevist $(\mathrm{N}=1,20 \mu \mathrm{mol} \mathrm{Gd} / \mathrm{kg})$. Vascular MRA at $1 \mathrm{~T}$ was performed (Aspect $\mathrm{M}^{\mathrm{TM}}, 500 \mathrm{mT} / \mathrm{m}, 2500 \mathrm{~T} / \mathrm{m} / \mathrm{s}$ ) using 3D-SPGR $(\mathrm{TR} / \mathrm{TE}=12 \mathrm{~ms} / 2.1 \mathrm{~ms}$, slice thickness $=1 \mathrm{~mm}$, FOV $=5 \mathrm{~cm}$, matrix $=128 \times 128, F A=45)$. Vessel wall MRI at $3 \mathrm{~T}$ was performed (Signa HDx, GE Healthcare, $50 \mathrm{mT} / \mathrm{m}$, $150 \mathrm{~T} / \mathrm{m} / \mathrm{s}$ ) with a phased-array mouse coil (RAPID MR International), using a double inversion recovery fast spin echo sequence $(\mathrm{TR} / \mathrm{TE}=400 \mathrm{~ms} / 15 \mathrm{~ms}$, slice thickness $=1 \mathrm{~mm}, \mathrm{FOV}=3 \mathrm{~cm}$, matrix $=256 \times 256)$ up to 24 hours after injection.
4) RGD-targeted P22 ex vivo fluorescence imaging: Molecular targeting of P22 was evaluated by attaching RGD peptides externally, which targets the $\alpha \mathrm{V} \beta 3$ integrin, upregulated on activated macrophages. $\mathrm{ApoE}^{-1-}$ mice $(\mathrm{N}=4)$ were injected intravenously with $\mathrm{RGD}^{+} \mathrm{P} 22$ or $\mathrm{RGD}^{-}$ P22 (labeled with Cy5.5, $4 \mathrm{nmol} /$ mouse). Forty-eight hours later, ex vivo fluorescence imaging was performed using Maestro ${ }^{\mathrm{TM}}$ imaging system (Cri, Woburn, MA). Maximum plaque signal intensities were measured and compared.

\section{Results}

Low dose P22-polymer-Gd showed strong enhancement for 1T vascular MRA (Figure 2). It also showed clear enhancement of the aortic wall $\left(\mathrm{ApoE}^{-1-}\right)$ and ligated carotid (FVB) at 3T (Figure 3). Ex vivo fluorescence imaging showed the accumulation of both $\mathrm{RGD}^{+} \mathrm{P} 22$ or RGD ${ }^{-} \mathrm{P} 22$ in atherosclerotic lesions (Figure 4). RDG targeting enhanced plaque uptake (RGD ${ }^{+}$22: $0.025 \pm 0.002$ counts/ sec vs. RGDP22: $0.005 \pm 0.004$ counts/sec, $\mathrm{p}=0.05$ ).

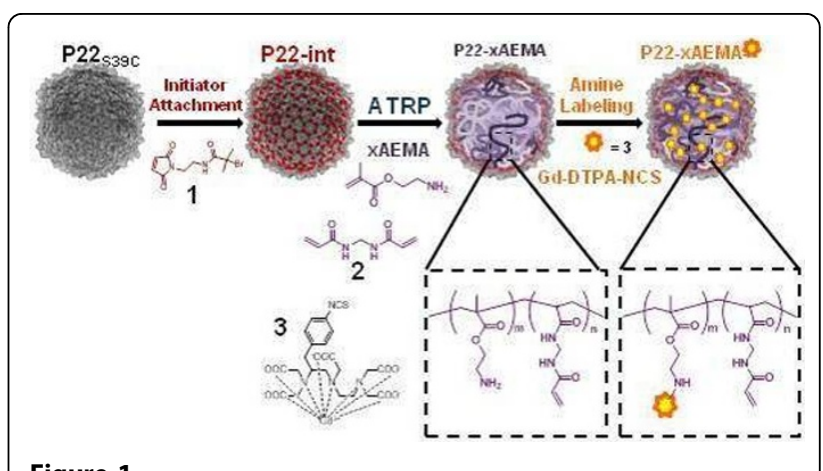

Figure 1

${ }^{1}$ Stanford University, Stanford, CA, USA

Full list of author information is available at the end of the article

(C) 2013 Kosuge et al; licensee BioMed Central Ltd. This is an Open Access article distributed under the terms of the Creative Commons Attribution License (http://creativecommons.org/licenses/by/2.0), which permits unrestricted use, distribution, and reproduction in any medium, provided the original work is properly cited. 


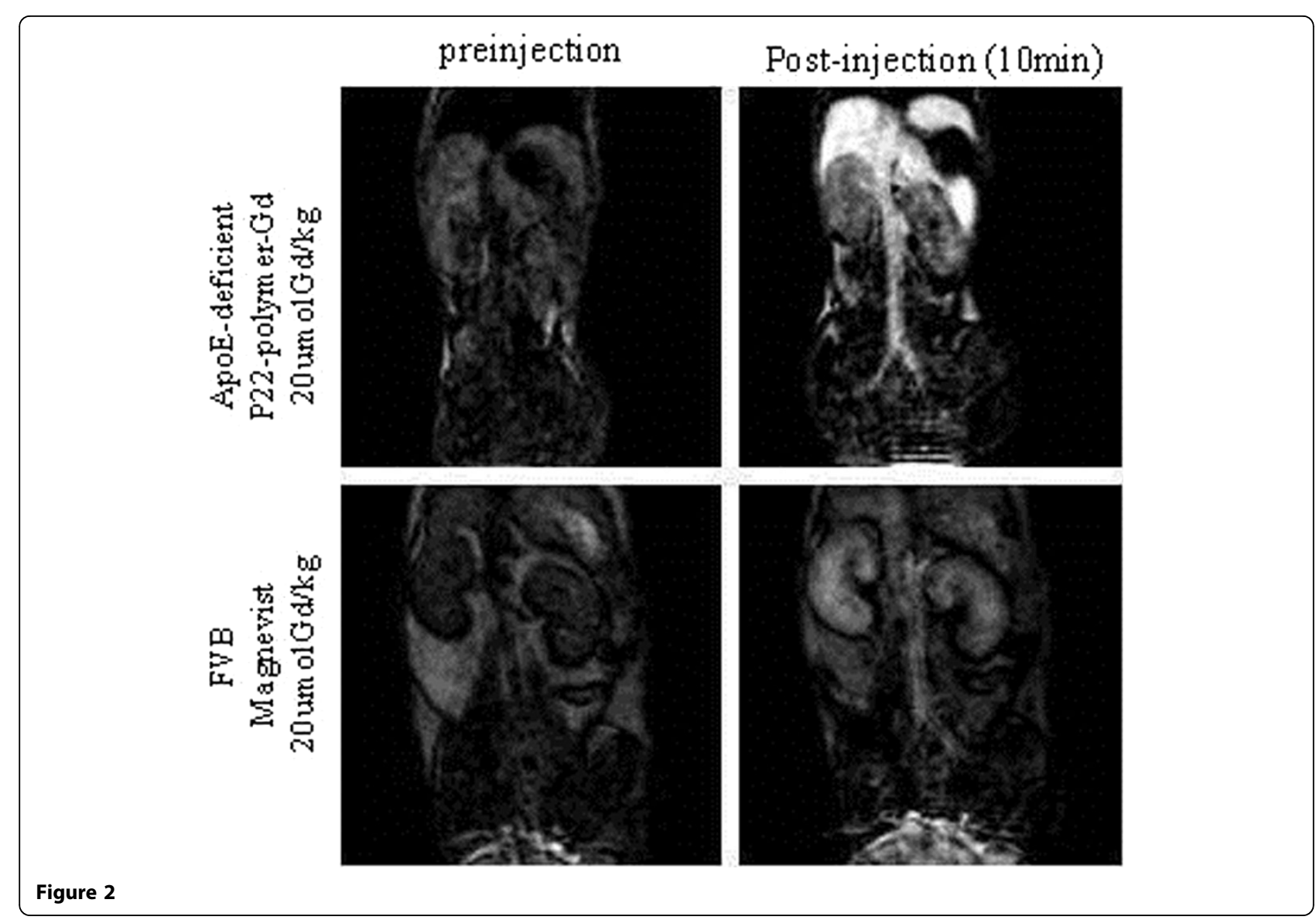

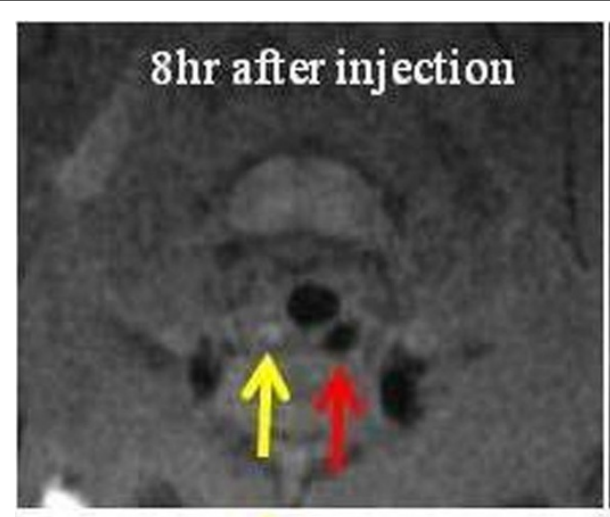

Left carotid

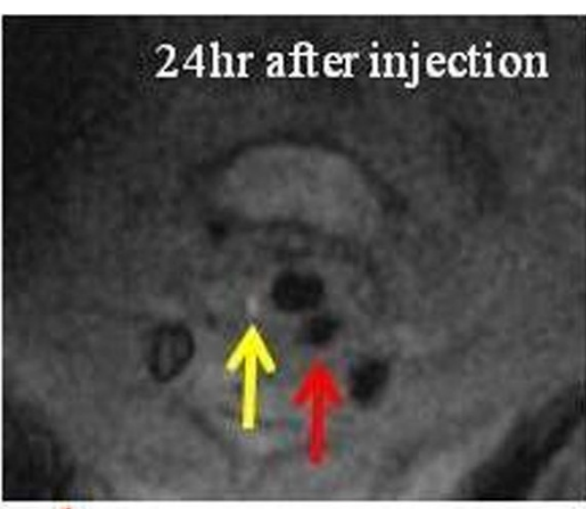

Right carotid

Figure 3

\section{Conclusions}

The P22 protein cage nanoparticle demonstrates both internal high-relaxivity Gd-loading for in vivo MRI as well as external RGD-targeting for enhanced uptake in vascular inflammation. Thus, P22 is a novel, multi-functional nanoparticle platform for targeted-imaging of atherosclerosis.

\section{Funding}

GE healthcare, Kowa, Inc.

Author details

${ }^{1}$ Stanford University, Stanford, CA, USA. ${ }^{2}$ Montana State University, Bozeman, MT, USA. 


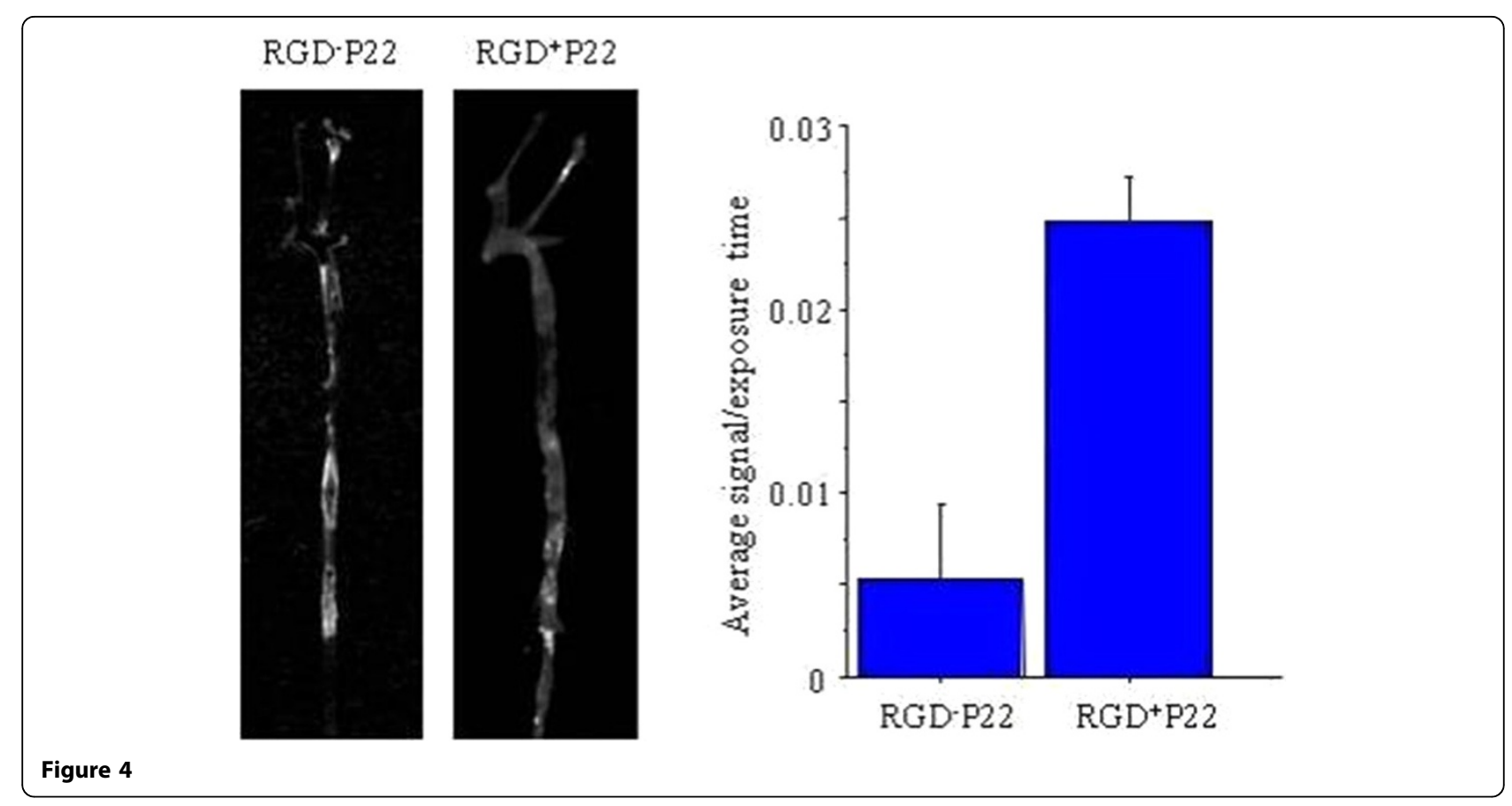

Published: 30 January 2013

\section{References}

1. Lucon J, et al: . Nat Chem 2012, 4:781-788.

2. Kosuge, et al: . PLoS One 2011, 6:e14523.

doi:10.1186/1532-429X-15-S1-066

Cite this article as: Kosuge et al:: High-Gd-Payload P22 protein cage

nanoparticles for imaging vascular inflammation. Journal of

Cardiovascular Magnetic Resonance 2013 15(Suppl 1):O66.

Submit your next manuscript to BioMed Central and take full advantage of:

- Convenient online submission

- Thorough peer review

- No space constraints or color figure charges

- Immediate publication on acceptance

- Inclusion in PubMed, CAS, Scopus and Google Scholar

- Research which is freely available for redistribution

Submit your manuscript at www.biomedcentral.com/submit 